\section{NO. 88}

FEBRUARY 2018

\section{ADB BRIEFS}

\section{KEY POINTS}

- Countries in Asia and the Pacific are investing in digital health for achieving universal health coverage (UHC), but they face challenges in doing so.

- Legal frameworks for data privacy and security are still nascent. Health informatics capacity needs strengthening, including understanding of health data use, health informatics standards, and of concepts such as interoperability and enterprise architecture.

- Most developing countries lack a robust land and civil registration system, which limits their ability to rely on existing registries for population groups, villages and administrative units to plan health services.

- Key issues to be addressed include understanding where the patients and the health services are; factors contributing to increased health risks for people; and how access to health services-both curative and preventive-can be improved in an expanding health care ecosystem.

- Effective management and use of geospatial data and technologies with support from the Health GeoLab Collaborative, can address the geographic aspects of pressing public health problems and improve planning of health services for UHC.

\section{BUILDING CAPACITY FOR GEO-ENABLING HEALTH INFORMATION SYSTEMS: SUPPORTING EQUITABLE HEALTH SERVICES AND WELL-BEING FOR ALL}

\section{Steeve Ebener \\ Data Management and GIS Consultant \\ Health GeoLab Collaborative}

\section{Susann Roth}

Senior Social Development Specialist

Sustainable Development and Climate Change Department

Asian Development Bank

\section{Sonalini Khetrapal}

Health Specialist

Sustainable Development and Climate Change Department

Asian Development Bank

\section{GEO-ENABLING HEALTH INFORMATION SYSTEMS}

Universal health coverage (UHC) - equitable access to quality health services without undue financial hardship-is the stated aim of almost every country in Asia and the Pacific. The effective management and use of geospatial data and technologies (GST, Box 1) support efforts in achieving UHC. They also support the exchange and use of information by systems interoperability, especially in countries with weak registration systems. They enable countries to address health system inefficiencies and inequities, and as such improve health service delivery, planning, and management for better use of health sector resources to leave no one behind. 


\section{Box 1: Geography, Geospatial Data, and Geospatial Technologies (GST)}

Geography: A science that deals with the description, distribution, and interaction of the diverse physical, biological, and cultural features of the earth's surface. ${ }^{\text {a }}$

Geospatial data: Also referred to as spatial data, information about the locations and shapes of geographic features and the relationships between them, usually stored as coordinates and topology.

Geospatial technologies: Refers to equipment used in visualization, measurement, and analysis of earth's features, typically involving such systems as global navigation satellite systems, geographical information systems, and remote sensing. ${ }^{c}$

Sources:

a Merriam-Webster. https://www.merriam-webster.com/dictionary/ geography.

b The GIS Encyclopedia. http://wiki.gis.com/wiki/index.php/GIS_Glossary.

c U.S. News and World Report. https://www.usnews.com/science/ articles/2011/05/11/geospatial-technology-as-a-core-tool.

Geospatial data is directly relevant to all three main functions of a country's public health system: monitoring community health and identification of health problems and priorities; ensuring universal access to appropriate and cost-effective care; and policy making to solve local and national health problems. Everything happens somewhere at a given time, yet space and time are two dimensions that are poorly captured in many health information systems (HIS).

The sophistication of GST can sometimes be a barrier, and it would be logical to assume in resource-constrained settings that lack of technology is the main obstacle to using it. This is not so. The significant barriers are related to geospatial data management practices, policies, and resources to ensure sustainability.

Among ADB's developing member countries (DMCs) few ministries of health have a data management unit. Moreover, neither strengthening of data management skills, nor improving the content of existing data systems, are particularly attractive to donor agencies deploying health-related funding, even when these funds are earmarked for health systems strengthening. What training there is tends to be sporadic, one-off sessions on the use of GST, which does not cover good and sustainable geospatial data management practices. Within governments, GST technical staff and health systems decision makers rarely have a meeting of minds, with the technical staff unable to convey to the decision makers that no amount of technology can overcome the limitations of lack of content and its quality. This is a fundamental issue with all digital health solutions.
A geo-enabled HIS provides powerful tools to address pressing public health problems and achieve UHC. It contextualizes data from different sources in both space and time using geographic objects (i.e., health facilities) as the common link between data collected by different sources using geography as the unifying element in data analysis. It also helps analyze trends in health data by taking into account changes in geography through time. Using GIS, an integrated collection of computer software and data can be used to view and manage information about places, analyze spatial relationships, and model spatial processes (Figure 1).

All of these facets are essential for malaria elimination, control of other communicable diseases, and health sector planning in general.

\section{THE HEALTH INFORMATION SYSTEM GEO-ENABLING FRAMEWORK}

The first step in harnessing the power of geospatial data and technologies to support a strong and effective health system is to have a geo-enabled HIS with the necessary elements in place. There are nine key elements of a geo-enabled health information systems that form the HIS geo-enabling framework initially developed and implemented by the Asia eHealth Information Network (AeHIN) GIS Lab:

(i) A clearly defined vision, strategy, and plan for geoenabling the HIS;

(ii) An information system governance structure covering geospatial data and technologies;

(iii) A minimum technical capacity to manage and use geospatial data and technologies;

(iv) Defined geospatial data specifications, standards and protocols to ensure the availability and quality of geospatial information across the whole data lifecycle;

(v) Master lists for the core geographic objects (e.g., health facilities, villages, administrative and reporting divisions) and their associated geography, with an updating mechanism in place for each through the use of a common georegistry;

(vi) Appropriate geospatial technologies identified in accordance with good geospatial data management practices;

(vii) Use cases supporting the implementation of programs towards reaching health-related Sustainable Development Goal (SDG) 3 and improving UHC;

(viii) Policies supporting and enforcing all of the above as well as geospatial data accessibility; and

(ix) The necessary resources to ensure sustainability over the long term. 
Figure 1. Uses of Geospatial Data

Contextualize data from different sources in both space and time

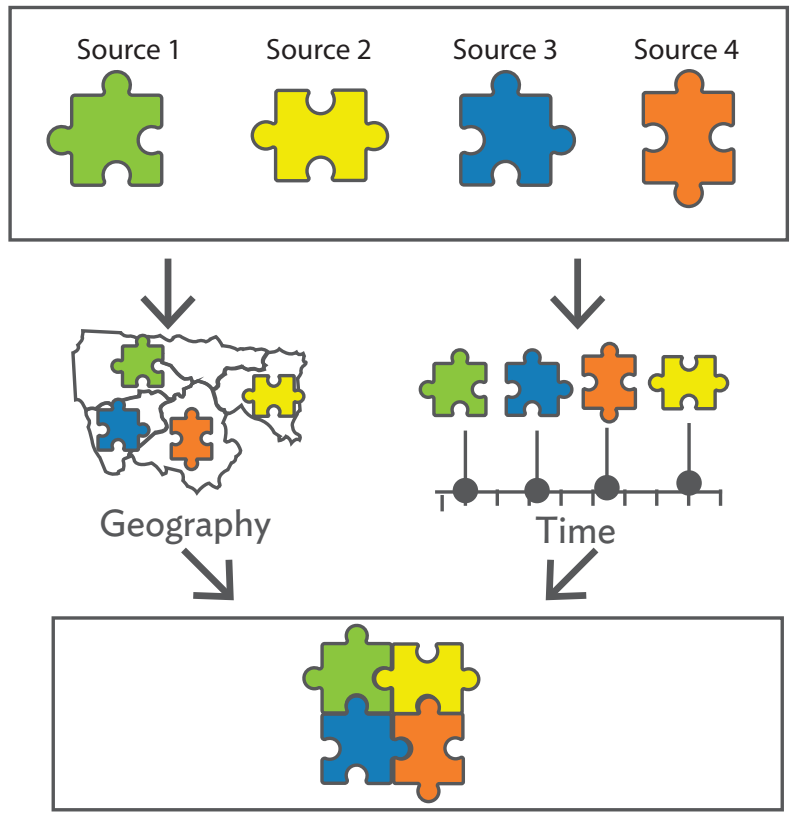

3

Facilitate trend analysis by taking into account how the geography has changed through time
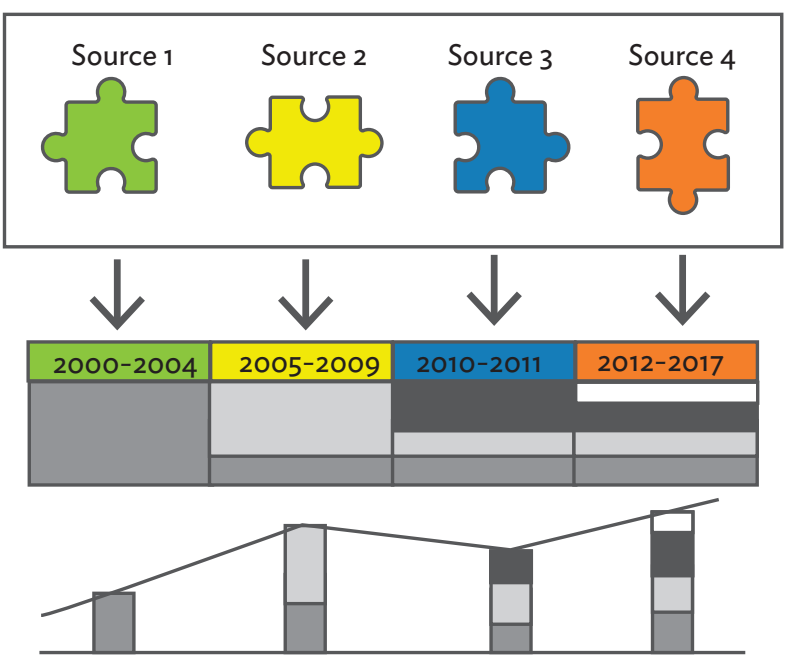

2 Use geographic objects (i.e. health facilities) as the common link between data collected by different sources
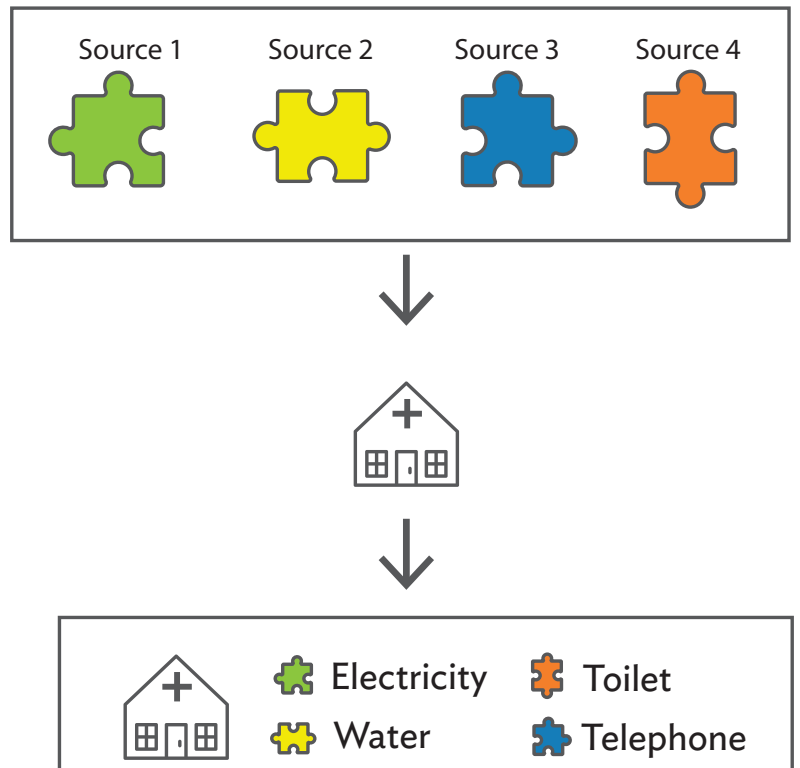


\section{GEO-ENABLING HEALTH INFORMATION SYSTEMS IN ASIA AND THE PACIFIC}

To better understand the current situation for countries in the region aspiring to geo-enable their HIS, in February 2017, the AeHIN GIS Lab, with ADB support, used the HIS geo-enabling framework to assess 13 countries aspiring to use geospatial data and technologies for their HIS, with the objective of informing the ministries of health and HIS decision makers (Figure 2).

While GIS technical capacity is present and has been used to create maps across all 13 countries, the results of the survey indicate important gaps for all the other seven components of the framework.
Almost all countries surveyed had no policy framework, and collected and used geospatial data that did not have the necessary specifications, standards, and protocols in place. Asked what they most needed to ensure the sustainability of their existing GIS programs, respondents typically cited training and capacity development. This illustrates the gaps that exist in the region to ensure a sustainable geo-enablement of the HIS.

\section{THE AeHIN GIS LAB-PROGRESS IN ONE YEAR}

To enable the health sector in countries to fully benefit from geospatial data and technologies, ADB and the World Health Organization (WHO), together with private sector partners, supported the establishment of a GIS laboratory under the umbrella of AeHIN. ${ }^{2}$

Figure 2. Situation Analysis of Health Information Systems Geo-Enabling in 13 Asia and the Pacific Countries

\begin{tabular}{|c|c|c|c|c|c|c|c|c|c|c|c|c|c|}
\hline Geo-enabling components & BAN & $\mathrm{BHU}$ & CAM & FIJ & IND & INO & LAO & MYA & MAL & NEP & $\mathrm{PHI}$ & SRI & VIE \\
\hline \multicolumn{14}{|l|}{$\begin{array}{l}\text { 1. Vision, needs, strategy, } \\
\text { and plan }\end{array}$} \\
\hline \multicolumn{14}{|l|}{ 2. Governance structure } \\
\hline \multicolumn{14}{|l|}{ 3. Technical capacity } \\
\hline \multicolumn{14}{|l|}{$\begin{array}{l}\text { 4. Data specifications, } \\
\text { standards, and protocols }\end{array}$} \\
\hline \multicolumn{14}{|l|}{$\begin{array}{l}\text { 5. Master list and common } \\
\text { georegistry }\end{array}$} \\
\hline \multicolumn{14}{|l|}{ 6. Geospatial technologies } \\
\hline \multicolumn{14}{|l|}{ 7. Map production (use) } \\
\hline \multicolumn{14}{|l|}{ 8. Policy } \\
\hline \multicolumn{14}{|l|}{$\begin{array}{l}\text { 9. Resources for } \\
\text { sustainability }\end{array}$} \\
\hline $\begin{array}{l}\text { BAN = Bangladesh, } \mathrm{BHU}=\text { Bhutan }, \mathrm{C} \\
\text { MYA = Myanmar, } \mathrm{MAL}=\text { Malaysia, } \mathrm{N} \\
\text { Source: Authors. }\end{array}$ & $\begin{array}{l}M=C \\
P=N\end{array}$ & isting & $\begin{array}{l}\text {, FIJ = } \\
=\text { Phili }\end{array}$ & $\ln \mathrm{p}$ & cess & $\mathrm{a}, \mathrm{VIE}$ & & lot exist & ting & & & & \\
\hline
\end{tabular}

HIS survey detailed findings can be found at AeHIN GIS Lab. 2017. Quick HIS Geo-Enabling Assessment Survey. www.healthgeolab.net/MEETINGS/NPT_2017/ Summary_quick_HIS_geo-enabling_assessment_survey_040317.pdf.

2 ADB. 2016. The Geography of Universal Health Coverage. ADB Briefs No. 55. Manila. http://www.adb.org/publications/geography-universal-health-coverage. 
Since its launch in June 2016, the AeHIN GIS Lab, has been using the 4Ts (Teaming, Tooling, Testing, Training) approach to strengthen in-country's capacity for proper and sustainable use of geospatial data and technologies for HIS.

In terms of teaming, the AeHIN GIS Lab has, for example, established a public-private partnership with Esri, the leading proprietary GIS software company, to allow ministries of health to access highly discounted GIS software bundles. Through ADB support, Cambodia, Myanmar, and Viet Nam have already benefited from such bundles. Esri is now revising the bundles to be even more affordable to countries in the region. The AeHIN GIS Lab has also established a network of peers with 152 members from 33 countries.

From a tooling and testing perspective, the AeHIN GIS Lab helped develop, test, and document version 5.0 of AccessMod ${ }^{3}$ a free toolbox developed by WHO to support UHC by modeling physical accessibility to health care. The Lab, in collaboration with partners such as the United Nations Children's Fund and Mahidol Oxford Research Unit, has released guidance documents to support the management and use of geospatial data in health.
On the teaming side, the AeHIN GIS Lab helped build regional capacity through workshops and also directly provided extensive onsite technical support in Cambodia, Myanmar, and Viet Nam for the geo-enablement of their HIS (Boxes 2 and 3).

\section{LOOKING AHEAD: THE HEALTH GeoLab COLLABORATIVE}

The AeHIN GIS Lab's activities in the region, especially in Cambodia, Myanmar, and Viet Nam, have helped countries to move forward with geo-enabling their HIS and harnessing the benefits of geospatial data and technologies. However, 27 other low- and middle-income countries in Asia and the Pacific could benefit from the same support.

The Health GeoLab Collaborative has been established to fill this gap in a cost-effective manner by bringing together more institutions and individuals sharing a common vision, for low- and middle-income countries in Asia and the Pacific to fully benefit from the power of GST to reach the health related SDG 3, and ready to engage their respective skills, experience, and resources to achieve it.

\section{Box 2. Case Study: Myanmar}

In Myanmar, the management and use of geospatial data and technologies directly contributes to the implementation of the National Health Plan (NHP) 2017-2021. The NHP 2017-2012 aims to strengthen Myanmar's health system, address its public health priority issues, and support the drive toward UHC. However, efforts to geo-enable the HIS were hampered by a lack of governance structure, standards, protocols and policy, and technical capacity. As a result, information systems were not using the same geographic data and therefore not fully benefiting from what geography, geospatial data, and technologies have to offer. A pilot project conducted in the Magway region was used to demonstrate the benefits of geo-enabling the HIS by using common master lists for the geographic objects core to public health (and thus the same geographic data) across three programs.

Under this project, ArcGIS mapping and AccessMod modeling software were used to propose a fully functional emergency obstetric care delivery network as a way to support planning. The unique health facility identifiers from the master list were integrated into the malaria database to visualize temporal changes in the number of positive malaria cases over 2015, as part of communicable disease monitoring and surveillance. The spatial analysis functions of ArcGIS were used to conduct a rapid impact assessment after the 2016 Chauk earthquake and thus illustrate how emergency management can be supported.
The implementation of the pilot project throughout the Magway region not only strengthened the technical capacity of the Ministry of Health and Sports in the management and use of geospatial data and technologies, but also demonstrated the benefits gained through geo-enabling the HIS:

- Duplication of efforts was avoided, which reduced costs through the use of common master lists and their associated geography;

- Quality (completeness, uniqueness, timeliness, validity, accuracy, and consistency) of geospatial data improved through the use of common guidelines, standards, and protocols;

- Geography was used as the dimension that connects data from different sources; and

- Powerful visualization and analytical capabilities of geographic information system technology were used (see "Geo-enabling the health information system in Myanmar story map" at go.esri.com/MOHS, for more details).

All the above support the implementation of the NHP 2017-2021 to reach the United Nations Sustainable Development Goals and is now being used to institutionalize what has been initiated through the pilot project as part of the strategic action plan for strengthening health information 2017-2021.

Source: Authors.

3 World Health Organization. Access Mod 5. www.accessmod.org. 


\section{Box 3. Case Study: Cambodia and Viet Nam}

The Asia eHealth Information Network (AeHIN) GIS Lab has been supporting Cambodia to strengthen the technical capacity of the Health Information System Bureau and the Center for Parasitology, Entomology, and Malaria Control in geospatial data management and technologies following an approach similar to that of Myanmar. In addition to implementing the pilot project aimed at demonstrating the benefits of geo-enabling the HIS and providing onsite and remote trainings, the Lab has facilitated inter-ministerial meetings to enhance the Ministry of Health Management Information System and Health Coverage Plan platform to become the common georegistry for the health sector in the country.

The geo-enabling process also started recently in Viet Nam. This process, which benefits from the experiences in both Cambodia and Myanmar, is not only strengthening the technical capacity of different entities with the Ministry of Health, including the National Institute of Malaria, Parasitology, and Entomology, but also setting the foundations for a suitable geo-enablement of the country's HIS.

Source: Authors.
The Health GeoLab Collaborative is being launched as the next natural iteration of the AeHIN GIS Lab to convene stakeholders, including government and partner agencies from the health sector in target countries, donor agencies, nongovernment organizations, academic institutions, and the private sector to drive future efforts. The Collaborative builds on the work done and the network of expertise developed through the activities of the AeHIN GIS Lab over 2016-2017.

In the coming years, the Collaborative is expected to become a reference, facilitator among stakeholders, and a knowledge broker in the region. It will help advocate the use of geography, geospatial data, and technologies to reach SDG 3, including UHC or key public health issues such as the passage from malaria control to malaria elimination.

The progress toward reaching this objective will be measured through indicators, including the amount of financial and in-kind support provided to countries to enable the management and use of geospatial data and technologies for their HIS, number of countries having geo-enabled their HIS; documentation of the benefits gained through the geo-enablement, and number of partners that have joined the Collaborative by 2025.
About the Asian Development Bank

ADB's vision is an Asia and Pacific region free of poverty. Its mission is to help its developing member countries reduce poverty and improve the quality of life of their people. Despite the region's many successes, it remains home to a large share of the world's poor. ADB is committed to reducing poverty through inclusive economic growth, environmentally sustainable growth, and regional integration.

Based in Manila, ADB is owned by 67 members, including 48 from the region. Its main instruments for helping its developing member countries are policy dialogue, loans, equity investments, guarantees, grants, and technical assistance.

ADB Briefs are based on papers or notes prepared by ADB staff and their resource persons. The series is designed to provide concise, nontechnical accounts of policy issues of topical interest, with a view to facilitating informed debate. The Department of Communications administers the series.
The views expressed in this publication are those of the authors and do not necessarily reflect the views and policies of ADB or its Board of Governors or the governments they represent.

Asian Development Bank

6 ADB Avenue, Mandaluyong City

1550 Metro Manila, Philippines

Tel +6326324444

Fax +6326362444

www.adb.org/publications/series/adb-briefs pubsmarketing@adb.org

Corrigenda to ADB publications may be found at http://www.adb.org/publications/corrigenda 